\title{
Laser-Induced Breakdown Spectroscopy for Wendelstein 7-X Stellarator Limiter Tile Analysis
}

\author{
Cong $\mathrm{Li}^{1,2}$, Niels Gierse ${ }^{2}$, Jannis Oelmann², Sebastijan Brezinsek ${ }^{2}$, Marcin Rasinski², Chandra Prakash \\ Dhard $^{3}$, Thomas Sunn Pedersen ${ }^{3}$, Ralf König ${ }^{3}$, Yunfeng Liang ${ }^{2}$, Hongbin Ding ${ }^{1}$, Christian Linsmeier ${ }^{2}$ and \\ the W7-X team $^{3}$
}

\author{
${ }^{1}$ Key Laboratory of Materials Modification by Laser, Ion and Electron Beams, Chinese Ministry of Education, \\ School of Physics, Dalian University of Technology, 116024 Dalian, P. R. China \\ ${ }^{2}$ Forschungszentrum Jülich GmbH, Institut für Energie- und Klimaforschung - Plasmaphysik, Partner of the \\ Trilateral Euregio Cluster (TEC), 52425 Jülich, Germany \\ ${ }^{3}$ Max-Planck-Institut für Plasmaphysik, 17491 Greifswald, Germany \\ Corresponding author: e-mail: c.li@fz-juelich.de (C. Li) and hding@dlut.edu.cn (H. Ding)
}

\begin{abstract}
Laser-Induced Breakdown Spectroscopy (LIBS) is a well-established elemental composition analysis method as well as one of the most promising candidates for in situ first wall diagnosis of fusion devices. In this work, limiter graphite tiles which were exposed in the initial operational phase (OP1.1) of the Wendelstein 7-X stellarator to He and $\mathrm{H}$ plasma are analyzed ex situ by LIBS employing a picosecond pulsed laser for the first time and compared with post mortem analysis techniques. Depth profiles of each element and 2D profile of the ratio of $\mathrm{H}$ and $\mathrm{C}$ atoms on the surface are investigated. Both $\mathrm{H}$ content and retention depth on the deposition dominated zone are higher than on the erosion dominated zone due to the formation of $\mathrm{C}$-H co-deposition layer. The results from LIBS are in agreement with those from the cross-sectional scanning electron microscopic image and electron dispersive X-Ray spectroscopy.
\end{abstract}

Keywords: laser-induced breakdown spectroscopy, plasma wall interaction, fuel retention, deposition, erosion

\section{Introduction}

Analysis and understanding of plasma wall interaction (PWI) such as material erosion, deposition, and fuel retention processes on plasma facing components (PFCs) are vital for the safe and reliable operation of long pulse nuclear fusion devices. For safety reason, the maximum quantity of tritium in the ITER vessel is limited to $700 \mathrm{~g}$ [1], which requires in situ monitoring of tritium retention. The codeposition formation on PFCs is accompanied with fuel retention [2,3] which is a key issue for fusion device, especially for long-pulsed operation fusion device such as ITER. Wendelstein 7-X (W7-X) [4] which is the biggest stellarator in the world has presented 3D plasma topology in its first operational 
campaign in limiter configuration with graphite-based PFCs [5]. Therefore, investigation of the role of $3 \mathrm{D}$ divertor concepts in the physics and control of edge transport and stabilities, heat and particle exhausts is essential [6]. Study on fuel retention and material migration of PFCs in W7-X is vital to understand the 3D PWI in stellarator. Laser-induced breakdown spectroscopy (LIBS) is a wellestablished elemental composition analysis method as well as one of the most promising candidates for in situ first wall diagnosis of fusion devices [7,8] which is essential for long-pulse operation as postmortem analysis is inhibited. Its capability has been recently demonstrated on measuring and monitoring the fuel retention and the composition of deposition layers on PFCs of several fusion devices such as EAST [9-11], JET [12,13], ASDEX [14] and TEXTOR [15].

In the present study, limiter graphite tiles which were exposed in the initial operational phase OP1.1 of the W7-X stellarator to He and H plasma were analyzed ex situ by LIBS. Picosecond pulsed laser was employed to reduce the thermal effect and increase the ablated depth resolution. 2D mapping and depth analysis of $\mathrm{H}$ retention on the surface were provided by LIBS and compared with other post mortem analysis techniques such as scanning electron microscope (SEM), focused ion beam (FIB) electron dispersive X-Ray spectroscopy (EDX) and thermal desorption spectroscopy (TDS). The comparison allows both the quantification of fuel retention as well as depth-resolved information in absolute scale with LIBS.

\section{Experimental Setup}

\subsection{Laboratory setup}

Schematic of LIBS setup in the lab is shown in Fig.1. W7-X limiter tile samples were mounted on an $X Y$ translation stage inside a vacuum chamber which was equipped with quartz windows. The vacuum chamber was pumped down to a pressure of $1 \times 10^{-5} \mathrm{~Pa}$ to reduce the effect from water in air. The light beam of a picosecond laser (EKSPLA, PL2241) with pulse duration of 35 ps were operated in $355 \mathrm{~nm}$ at $10 \mathrm{~Hz}$. The laser beam was focused on the sample surface by a lens with a focal length of 500 $\mathrm{mm}$. By focusing the laser beam to a diameter of $0.9 \mathrm{~mm}$ and laser energy of $20 \mathrm{~mJ}$, the laser energy density of around $4 \mathrm{~J} / \mathrm{cm}^{2}$ was achieved. The optical emission of plasma produced by laser was collected in a direction of $45^{\circ}$ to the normal direction of sample surface by optical lens with diameter of $100 \mathrm{~mm}$ and focal length of $300 \mathrm{~mm}$. The LIBS signal light was transported by a single core fiber with diameter of $600 \mu \mathrm{m}$ and numerical aperture of 0.22 into a USB spectrometer with wavelength from 350 to 800 nm (Ocean Optics, HR2000). A spectral resolution of about $1 \mathrm{~nm}$ (FWHM) was obtained. SEM, FIB, EDX and TDS were also used for post mortem analysis. 


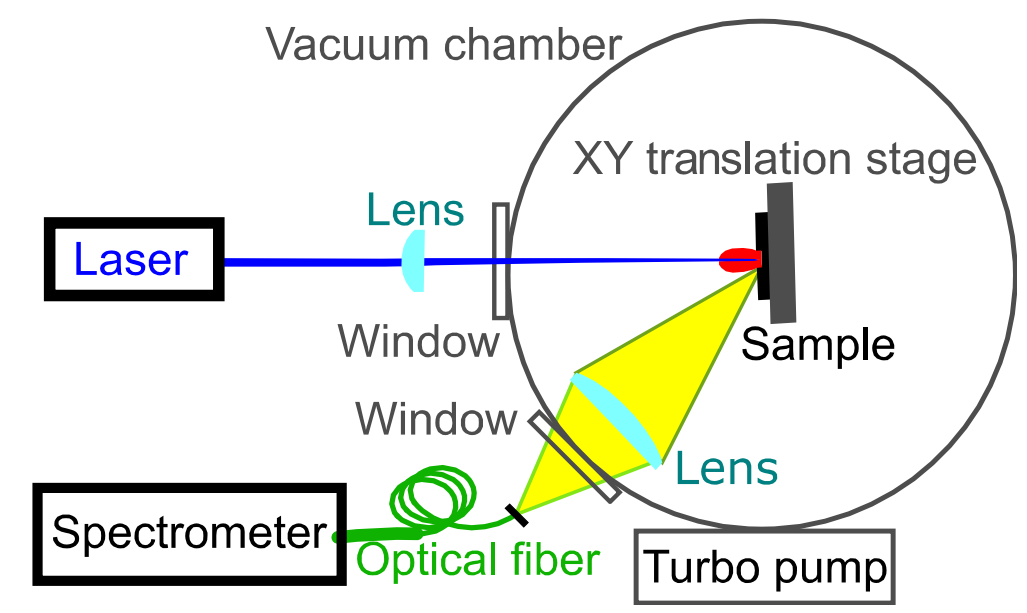

Fig.1 Schematic of ps LIBS setup with vacuum chamber for W7-X limiter tile sample measurement.

\subsection{Sample}

During the first operational phase (OP1.1) of the W7-X, five specially shaped poloidal limiters, each consisting of nine discrete graphite tiles were used as the main PFCs [16]. Limiters were taken out immediately after the initial operational phase of $311 \mathrm{~s}$ stellarator plasma exposure [17]. The graphite tile number 6 of limiter number 4 was measured in this work. Dual erosion/deposition stripes were noteworthy on the surface of the tile (Fig.2). According to Winters's study [18], from the center to the edge of the tile along toroidal direction, four basic features were found: a smoothened center region, a rough deposit region, a pure erosion region and a smooth deposition region. With our measurement, we studied erosion dominated zone near the center of the tile where tile received high flux plasma exposure and deposition dominated zone near the edge of the tile where $\mathrm{C}$ was re-deposited from erosion zone. LIBS was measured along lateral $(x)$ from the middle of tile $(x=0 \mathrm{~mm})$ to the edges of curved surface. 42 positions with two lines along lateral $(x)$ direction were measured by LIBS. The middle of tile was set as $x=0 \mathrm{~mm}$ position. The LIBS measurement at the left edge and right edge of curved surface were at the positions of $x=-96 \mathrm{~mm}$ and $x=96 \mathrm{~mm}$, respectively. Depth profile was obtained by consecutive laser pulses on each LIBS measurement position. In addition, reference sample which was spare limiter tile without using and plasma exposure was used to compare to the used tiles. 


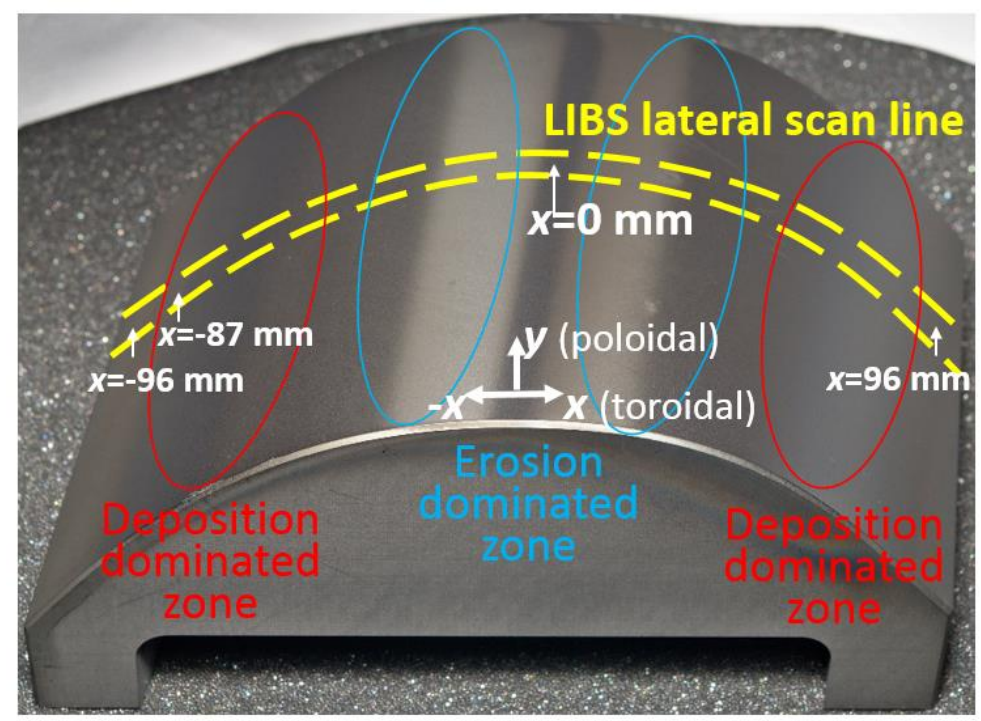

Fig.2 Photo of W7-X limiter tile exposed in OP.1.1. LIBS later scan line show the spatial resolved LIBS measurement along later direction.

\section{Results}

\subsection{Cross-sectional on sample surface}

Fig.3a shows the cross-sectional SEM image by FIB cutting on an erosion dominated zone of the sample on porous graphite. Due to high flux plasma exposure, there was no evidence of deposition on the erosion dominated zone. However, on a deposition dominated zone (Fig.3b), a smooth deposition layer with a thickness between 680 to $1100 \mathrm{~nm}$ on top of the graphite substrate was found.

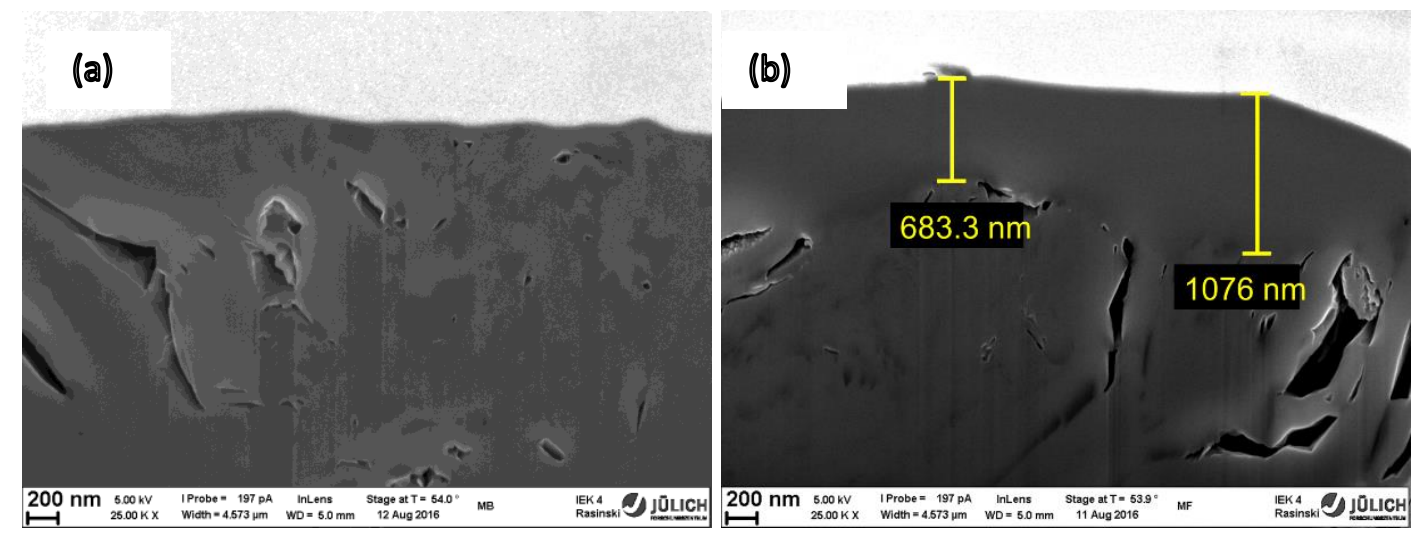

Fig. 3. Cross-sectional SEM images of (a) erosion dominated zone and (b) deposition layer with a thickness between 680 to $1100 \mathrm{~nm}$ on W7-X limiter tile. 
Fig.4 shows the typical LIBS spectra of $1^{\text {st }}$ and $10^{\text {th }}$ laser shot on the same position $(x=-96 \mathrm{~mm})$ on the sample. $\mathrm{H}(656.3 \mathrm{~nm}), \mathrm{Na}(589.0 \mathrm{~nm}), \mathrm{Fe}(396.9 \mathrm{~nm})$ atomic lines and $\mathrm{C}$ ionic $(657.8 \mathrm{~nm})$ lines were used for LIBS analysis. All these elements signals were found on the $1^{\text {st }}$ laser shot spectrum, however only $\mathrm{C}$ as substrate signal was found on the $10^{\text {th }}$ shot spectrum. For depth profile analysis, the single shot LIBS spectra were recorded for 15 successive laser shots at the same position ( $x=-87 \mathrm{~mm})$. Fig.5 shows that the intensity of the $\mathrm{H}, \mathrm{Na}$ and $\mathrm{Fe}$ peaks decreased with the number of laser shots and vanished within a few laser shots (few hundreds of $\mathrm{nm}$ ). Laser ablated depth was achieved by measuring crater profile by profilometer. EDX depth measurement was also used to calibrate the depth profile of LIBS on deposition layer. Two measurements showed an agreement result that about $100 \mathrm{~nm}$ ablated depth per laser under laser energy of $4 \mathrm{~J} / \mathrm{cm}^{2}$. Na signal was much higher than what it was in the reference sample. Due to very low limit of detection of LIBS for Na element ( ppb), Na as impurity in the sample should be very low content. Fe element might come from the impurity transport from steel PFCs in W7$\mathrm{X}$ during discharging. The $\mathrm{C}$ substrate was the only element whose intensity increased with the laser pulse and then plateaued.



Fig.4 Typical LIBS spectra of 1st and 10th laser on the same position $(x=-96 \mathrm{~mm}), \mathrm{H}, \mathrm{Na}, \mathrm{Fe}$ atom lines and $\mathrm{C}$ ionic lines were found. 


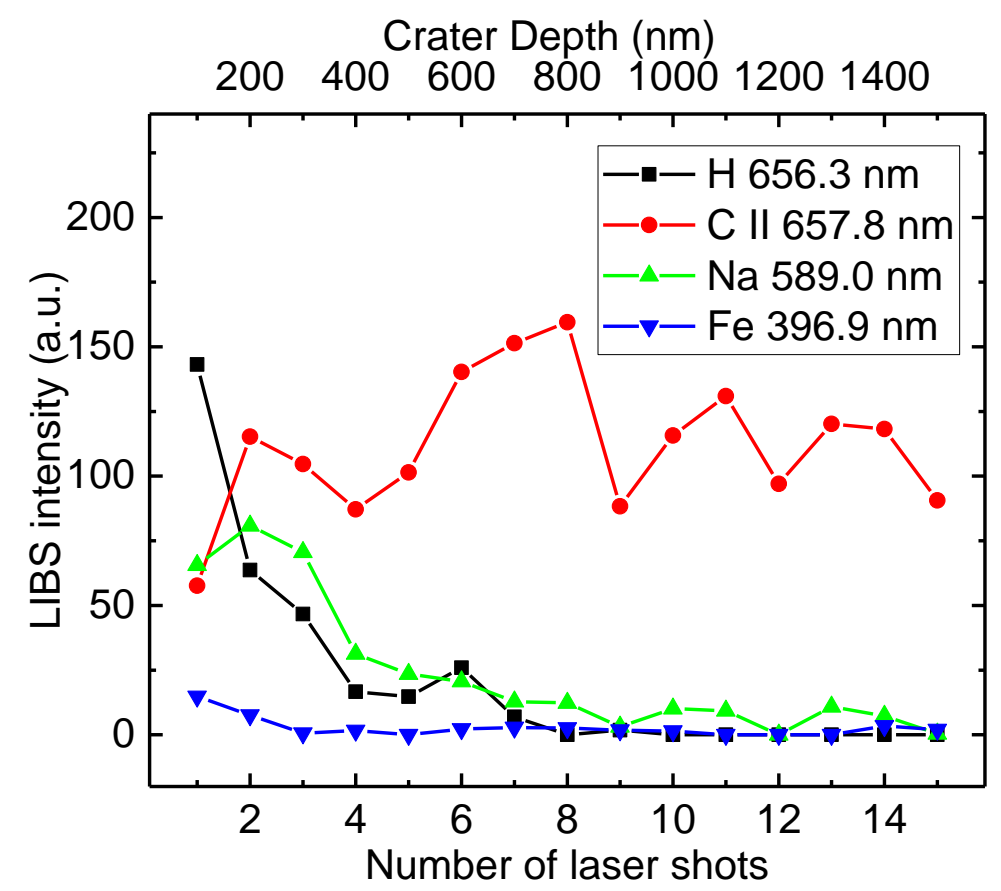

Fig.5 Depth profiles of $\mathrm{H}, \mathrm{C}, \mathrm{Na}$ and Fe elements on deposition dominated zone $(x=-87 \mathrm{~mm})$ by laser energy density of $4 \mathrm{~J} / \mathrm{cm}^{2}$.

Fig.6 shows a lateral profile of the ratio of $\mathrm{H}$ and $\mathrm{C}$ atoms by integrating signal of 10 laser ablations. Total of 42 points were considered in two vertical ( $y$ direction) lines along the curved surface to get LIBS spectra for 2D elemental mapping analysis. A spare limiter tile without using and plasma exposure was used as reference sample to compare to the used tile. Absolute amount of $\mathrm{H}$ in the tile was obtained by TDS measurement as well as Short Pulse Laser Ablation Gas analysis (SPLAG) [19]. Then absolute amount density of $\mathrm{H}$ was used to calibrate the LIBS $\mathrm{H}$ signal. Laser ablated volume on $\mathrm{C}$ per laser pulse was about $6.36 \times 10^{-5} \mathrm{~mm}^{3}$ by measurement of profilometer. Assuming the density was same in the sample, the number of $\mathrm{C}$ atom per laser ablation of $5.85 \times 10^{16}$ can be obtained by using bulk density of $1.83 \mathrm{~g} / \mathrm{cm}^{3}$. Due to $\mathrm{H}$ plasma exposure, $\mathrm{H}$ contents were between 0.14 and 0.4 in the limiter tile. These were obviously higher than $\mathrm{H}$ content in the reference sample without plasma exposure. The lateral trend was almost symmetrical and consistent with stripes on the surface of the tile in Fig.2. Due to the formation of $\mathrm{C}-\mathrm{H}$ co-deposition layer, $\mathrm{H}$ content in deposition dominated zone was higher than in the middle part (erosion dominated zone) of the tile where $\mathrm{H}$ was implanted due to high flux plasma exposure. $\mathrm{H}$ content at the edge of the tile far away from the plasma exposure zone was lower than in any other zone on the tile. 


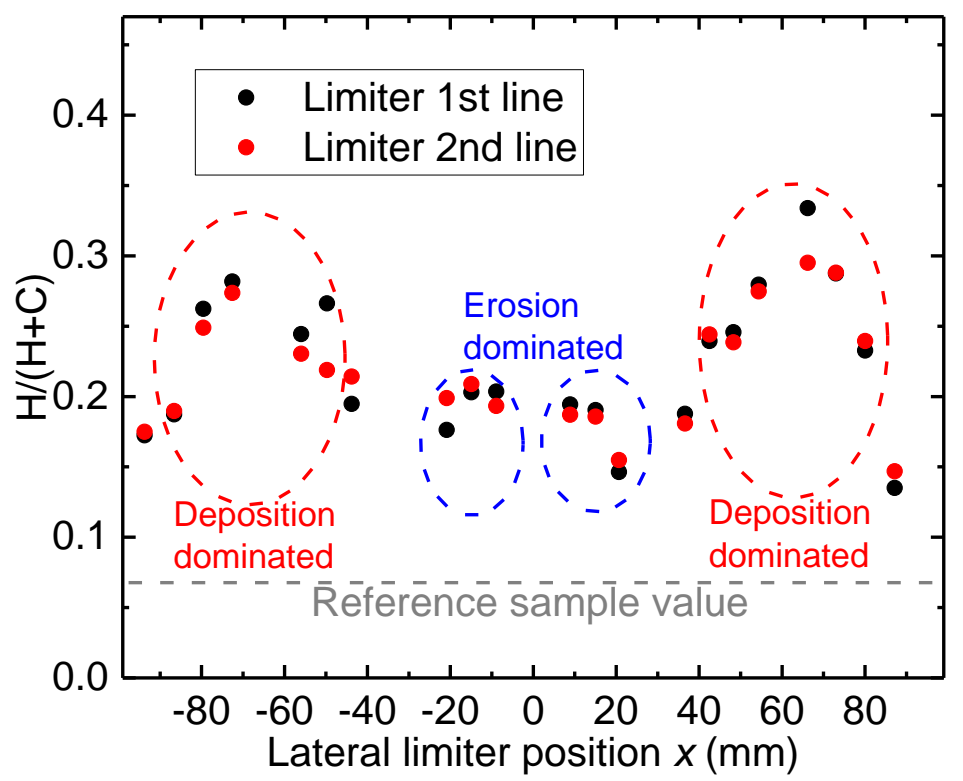

Fig.6 Lateral profile of the ratio of $\mathrm{H}$ and $\mathrm{C}$ atoms with different positions $(x)$, about $0.08 \mathrm{H}$ content was found in the reference sample without plasma exposure.

Fig.7 shows the depth profile of $\mathrm{H}$ content on the different zones on samples. $\mathrm{H}$ content was always very high on the top of the surface in the first laser shot due to contamination from air. $\mathrm{H}$ retention in deposition dominated zone existed about 10 laser shots (1000 nm depth) which was much deeper than in erosion dominated zone where $\mathrm{H}$ retention existed only for few laser shots. The thickness of C-H codeposition layer was around $1000 \mathrm{~nm}$ by LIBS measurement which was consistent with the cross-section result in Fig.3. On the reference sample without plasma exposure, most of H retention existed on the top layer of surface and there was almost no $\mathrm{H}$ content from the second laser shot. EDX analysis with $\mathrm{C}$ and $\mathrm{O}$ intensity on the different zones on the sample is shown in Fig.8. On erosion dominated zone, no $\mathrm{O}$ layer was found. There was clear O layer with thickness of about $900 \mathrm{~nm}$ on deposition dominated zone. This means that a C-H-O mixed deposition layer may be formed on the deposition dominated zone on the limiter tile. However, O concentration in the sample was below the limit of detection of the LIBS setup. The thickness analyses of $\mathrm{C}$ by LIBS and $\mathrm{O}$ by EDX were in agreement. 


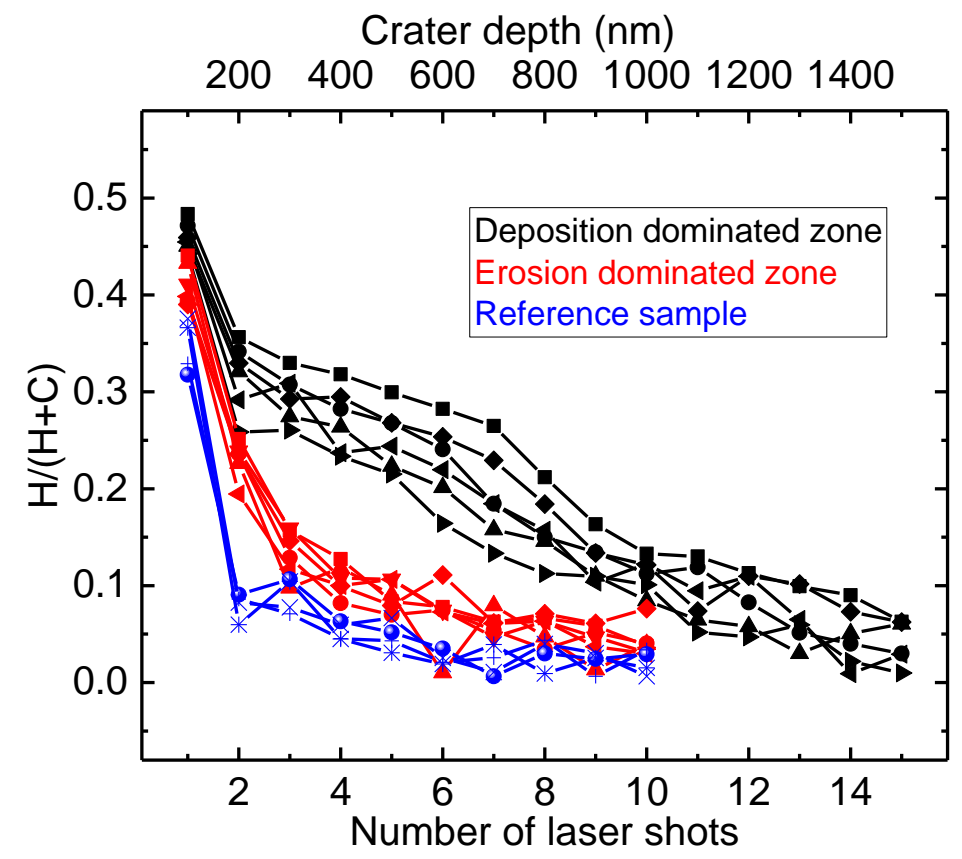

Fig.7 Depth profile of the ratio of $\mathrm{H}$ and $\mathrm{C}$ atoms on deposition dominated zone, erosion dominated zone and reference sample without plasma exposure by laser energy density of $4 \mathrm{~J} / \mathrm{cm}^{2}$.

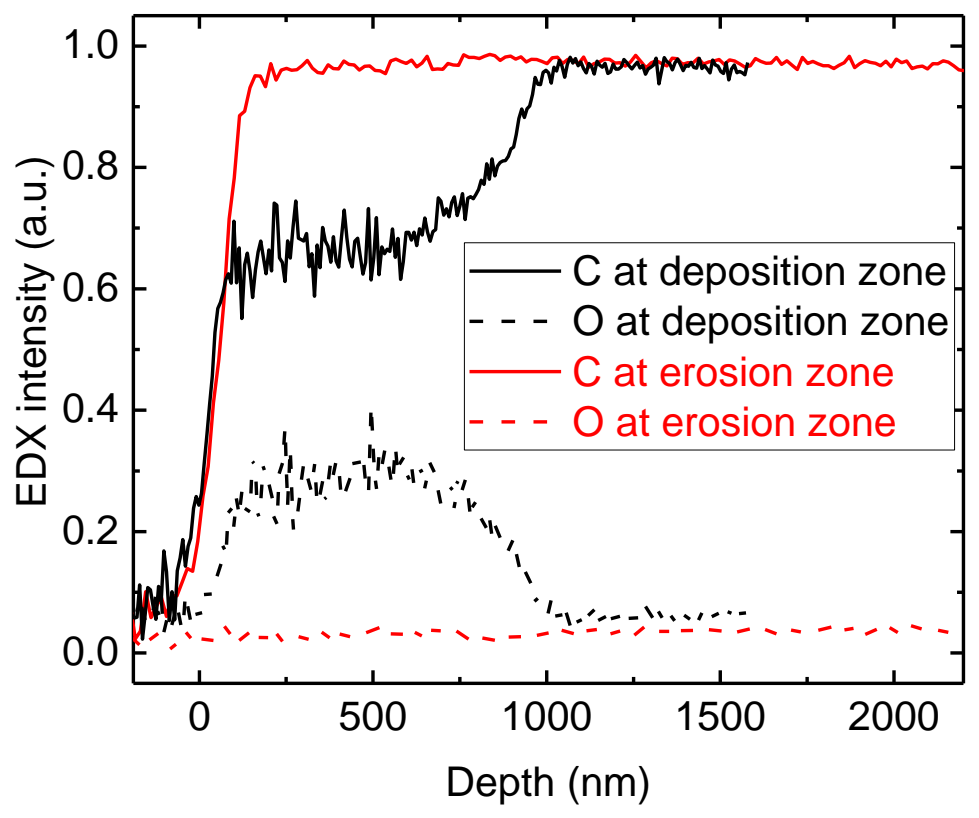


Fig.8 Depth profile of EDX intensity of $\mathrm{C}$ and $\mathrm{O}$ on deposition dominated zone and erosion dominated zone. A clear O layer with thickness of about $900 \mathrm{~nm}$ was found on deposition dominated zone

\section{Conclusion}

In this work, limiter graphite tiles which were exposed in the OP1.1 of the W7-X to He and $\mathrm{H}$ plasma were analyzed ex situ by LIBS employing a ps pulsed laser and compared with post mortem analysis techniques. A smooth deposition layer with thickness between 680-1100 nm could be found on the deposition dominated zone on the sample by cross-sectional SEM images. Elemental depth analysis shows that $\mathrm{H}, \mathrm{Na}$ and $\mathrm{Fe}$ peak intensities decreased with the number of laser shots and vanished within a few laser shot (few hundreds of $\mathrm{nm}$ ). Impurity Na signal was much higher than what it was it in the reference sample without plasma exposure. Fe element might come from the impurity transport from steel PFCs in W7-X during discharging. 2D profile of the ratio of $\mathrm{H}$ and $\mathrm{C}$ atoms shows that $\mathrm{H}$ content on the used tile was higher than what it was on the reference sample without plasma exposure. Due to the formation of $\mathrm{C}-\mathrm{H}$ co-deposition layer, the $\mathrm{H}$ content on deposition dominated zone was much higher than in the erosion dominated zone. A maximum ratio of $\mathrm{H}$ and $\mathrm{C}$ atoms could reach up to 0.4 on the deposition dominated zone. The $\mathrm{H}$ content of the reference sample without plasma exposure was only around 0.08. LIBS depth analysis shows the deposition layer was around $1000 \mathrm{~nm}$ on the deposition dominated zone. The $\mathrm{H}$ retention on the erosion dominated zone was only few hundreds of $\mathrm{nm}$. EDX depth analysis shows deposition layer with thickness of about $900 \mathrm{~nm}$, consistent with LIBS and SEM results. In situ study on the mapping of the PFCs surface by LIBS will be crucial to understand the 3D plasma on the plasma wall interaction in the stellarator geometry for further operation phases.

\section{Acknowledgements}

This work was supported by the National Magnetic Confinement Fusion Science Program of China (No.2013GB109005), National Science Foundation of China (Nos.11475039, 11605023), China Postdoctoral Science Foundation (Nos.2016M591423, 2017T100172) and the Fundamental Research Funds for the Central Universities (Nos.DUT15RC(3)072, DUT16TD13). This work has been carried out within the framework of the EUROfusion Consortium and has received funding from the Euratom research and training programme 2014-2018 under grant agreement No 633053. The views and opinions expressed herein do not necessarily reflect those of the European Commission.

\section{References}

[1] Roth J et al 2009 J. Nucl. Mater. 390-391 1-9. 
[2] Rubel M et al 2001 J. Nucl. Mater. 290-293 473-477.

[3] Brezinsek S et al 2013 Nucl. Fusion 53083023.

[4] Bosch H -S et al 2016 Nucl. Fusion, IAEA Fusion Energy Conference.

[5] Wurden G A et al 2017 Nucl. Fusion 57056036.

[6] Liang Y et al 2017 Nucl. Fusion 57066049.

[7] Philipps V et al 2013 Nucl. Fusion 53093002.

[8] Li C et al 2016 Front. Phys. 11114214.

[9] Li C et al 2015 J. Nucl. Mater. 463 915-918.

[10] Hu Z et al 2017 Plasma Sci. Technol. 19025502.

[11] Hai R et al 2014 J. Nucl. Mater. 438 S1168-S1171.

[12] Semerok A et al 2016 Spectrochim. Acta Part B 123 121-128.

[13] Karhunen J et al 2015 J. Nucl. Mater. 463 931-935.

[14] Paris P et al 2015 Fusion Eng. Des. 98-99 1349-1352.

[15] Xiao Q et al 2015 J. Nucl. Mater. 463 911-914.

[16] Sunn Pedersen T et al 2015 Nucl. Fusion 55126001.

[17] Wauters T et al 2016 In Poster Presented at 43rd EPS Conference on Plasma Physics, Leuven.

[18] Winters V R et al 2017 PFMC conference, Neuss.

[19] Oelmann J et al 2017 In Poster Presented at PFMC conference, Neuss. 